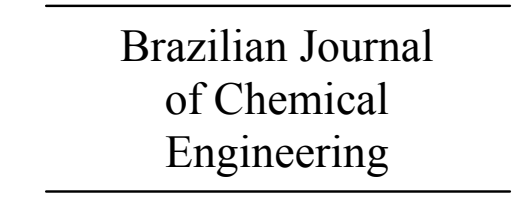

ISSN 0104-6632

Printed in Brazil

www.abeq.org.br/bjche

Vol. 31, No. 01, pp. 1 - 8, January - March, 2014

\title{
EXPRESSION OF Saccharomyces cerevisiae $\alpha$-GLUCOSIDE TRANSPORTERS UNDER DIFFERENT GROWTH CONDITIONS
}

\author{
S. L. Alves Jr. ${ }^{1,3^{*}}$, J. M. Thevelein ${ }^{3,4}$ and B. U. Stambuk ${ }^{2}$ \\ ${ }^{1}$ Universidade Federal da Fronteira Sul, Campus Chapecó, Unidade Bom Pastor, Sala 1-2-11, \\ Phone: + (55) (49) 2049-3106, Av. Fernando Machado 108-E, CEP 89802-112, Chapecó - SC, Brazil. \\ E-mail: slalvesjr@uffs.edu.br \\ ${ }^{2}$ Departamento de Bioquímica, Universidade Federal de Santa Catarina, Florianópolis - SC, Brazil. \\ ${ }^{3}$ Laboratory of Molecular Cell Biology, Institute of Botany and Microbiology, Katholieke Universiteit Leuven, and \\ ${ }^{4}$ Department of Molecular Microbiology, VIB, Kasteelpark Arenberg 31, B-3001, \\ Leuven-Heverlee, Flanders, Belgium.
}

(Submitted: February 28, 2012 ; Revised: April 17, 2013 ; Accepted: June 12, 2013)

\begin{abstract}
Important biotechnological processes depend on the efficient fermentation by Saccharomyces cerevisiae yeasts of starch hydrolysates rich in maltose and maltotriose. The rate-limiting step for fermentation of these $\alpha$-glucosides is the transport across the plasma membrane of the cells. In order to contribute to a better understanding of maltose and maltotriose metabolism by $S$. cerevisiae, we analyzed the expression of the main $\alpha$-glucoside transporter genes in two different yeast strains grown on media with glucose, maltose or maltotriose as carbon source. Although both yeast strains have higher $\alpha$-glucoside transport activity during growth on maltotriose, our results show similar expression levels of the analyzed genes on either maltose or maltrotriose media. Thus, our results indicate that, although the transport capacity of maltotriose grown cells is higher than that of maltose grown cells, maltotriose cannot be considered a better inducer of $\alpha$-glucoside transporter genes.
\end{abstract}

Keywords: Expression; Transporters; MAL genes; Maltose; Maltotriose; S. cerevisiae.

\section{INTRODUCTION}

Humans have been using yeasts in different biotechnological processes, such as baking and brewing, for millennia. Saccharomyces cerevisiae is considered to be the predominant agent in these processes, although other close Saccharomyces species (and hybrids among them) have also been isolated from these industrial applications (Querol and Bond, 2009). Baking and brewing rely on the efficient fermentation by yeasts of starch hydrolysates rich in maltose and maltotriose. In the brewing industry, for example, these two sugars are of special importance since they are the predominant sugars in wort (gener- ally $50-60 \%$ is maltose and $15-20 \%$ is maltotriose), followed by glucose (10-15\%) and other minor carbohydrates (Willaert, 2001). Of these sugars, glucose is preferentially and rapidly utilized by yeast cells, but both process efficiency and product quality require the complete fermentation of all sugars, including maltose and maltotriose. Although maltose is easily fermented by the majority of yeast strains after glucose exhaustion, maltotriose is not only the least preferred sugar for uptake by these Saccharomyces cells, but many yeasts will not use this $\alpha$-glucoside at all (Zheng et al., 1994; Yoon et al., 2003; Duval et al., 2010). Slow and incomplete yeast sugar fermentation represents a significant

\footnotetext{
*To whom correspondence should be addressed

This is an extended version of the manuscript presented at the SINAFERM 2011 -

18th National Symposium on Bioprocesses 2011, Caxias do Sul, Brazil.
} 
economic loss for these industries, and consequently most strain development programs aim to select yeasts with improved fermentation performance.

Utilization of maltose and maltotriose by yeast cells initially requires their active transport across the plasma membrane by $\mathrm{H}^{+}$symporters (Han et al., 1995; Alves et al., 2007, 2008). In the cytoplasm, these sugars are hydrolyzed by $\alpha$-glucosidases (maltases), releasing glucose molecules which will be metabolized through glycolysis to ethanol (Zastrow et al., 2001; Novak et al., 2004). Previous genetic and biochemical studies focusing mainly on the maltose fermentation system revealed a series of five unlinked telomere-associated $M A L$ loci: $M A L 1$ through MAL4 and MAL6. Each locus contains at least one copy of three different genes encoding a maltose permease (MALx1, where $x$ stands for one of the five $M A L$ loci mentioned above) responsible for the active uptake of the sugar, an intracellular maltase or $\alpha$-glucosidase (encoded by $M A L \times 2$ ), and a positive regulatory protein (encoded by $M A L \times 3$ ) that induces the transcription of the two previous genes in the presence of maltose. In order to be able to ferment maltose, a $S$. cerevisiae strain needs to have only one copy of these three genes (Needleman, 1991; Novak et al., 2004). These genes present in the different $M A L$ loci show a high degree of sequence and functional homology, but there may also be extensive variability since these genes are telomeric and several different alleles that determine distinct phenotypes (i.e., $M A L$-inducible and $M A L$-constitutive phenotypes) have been described previously. The MAL1 locus is considered to be the progenitor locus from which all other $M A L$ loci were derived, as all $S$. cerevisiae strains contain $M A L 1$ sequences at chromosome VII. This pattern holds true even for many non-maltose-fermenting yeast strains, which may harbor partially functional mallp (MAL13 mal11 mal12), mallg (mal13 MAL11 MAL12), or mal1 $^{0}$ (mal13 mal11 MAL12) loci containing just a functional regulator, just a maltose permease and maltase genes, or just a maltase gene, respectively (Naumov et al., 1994, Han et al., 1995). More recently, two other highly homologous genes (MPH2 and $M P H 3$ ) were described as encoding for maltose and maltotriose transporters. $M P H 2$ and $M P H 3$ are closely related to the MALx 1 genes, and apparently also induced by maltose (Day et al., 2002), although their role in maltotriose uptake and fermentation has been a matter of debate (Alves et al., 2008; Duval et al., 2010).

Maltose transport into the cell is required for full induction of $M A L$ genes, and several reports have shown that maltose uptake is also the rate-limiting step for fermentation (Kodama et al., 1995; Wang et al., 2002; Rautio and Londesborough, 2003). In the presence of maltose, the MALx3 regulatory protein binds to DNA at the promoter region between MALx1 and MALx2 genes (the so-called $\mathrm{UAS}_{\mathrm{MAL}}$ sequence), inducing the transcription of the MALx 1 transporter and MALx 2 maltase genes present at any $M A L$ locus (Michels and Needleman, 1984; Needleman et al., 1984; Kim and Michels, 1988; Levine et al., 1992; Sirenko et al., 1995). In the case of maltose, an increased flow of this sugar into the cells and its subsequent accumulation in the cytoplasm can act as a signal to induce higher expression of $M A L$ genes and to allow fermentation of this carbon source. Indeed, it was shown that both transport activity and levels of intracellular maltose are directly related to levels of induction of $M A L$ genes (Wang et al., 2002).

On the other hand, the presence of glucose can repress the expression of $M A L$ genes in two different ways: (1) inhibiting the activation of the regulatory protein $M A L x 3$ by maltose and (2) activating the $M I G 1$ repressor, which prevents the transcription of the three $M A L$ genes by binding at their promoter regions (Hu et al., 1995, 2000; Santangelo, 2006). In addition to its role in regulating transcription of $M A L$ genes, glucose also exerts a strong control on the fermentation of other carbon sources by promoting the inactivation of their respective transport systems. This catabolic inactivation involves the phosphorylation and ubiquitination of the transporters, followed by their endocytosis and vacuolar degradation (Riballo et al., 1995; Medintz et al., 1996).

Nevertheless, some yeast strains express their $M A L$ genes with no need of the induction by maltose. This phenotype is found in the so-called $M A L$ constitutive $\left(M A L^{C}\right)$ strains. The $M A L^{C}$ alleles are usually the result of mutations on the genes that encode the regulatory $M A L x 3$ protein. It is known that, when maltose binds to $M A L x 3$, this regulatory protein changes its structure and then is able to bind at the $\mathrm{UAS}_{\mathrm{MAL}}$ region of $M A L$ genes. However, in the case of constitutive MALx3 alleles, the mutations promote a different structure of the protein, allowing it to bind at the $\mathrm{UAS}_{\mathrm{MAL}}$ region even in the absence of maltose (Wang and Needleman, 1996; Gibson et al., 1997). In addition, $M A L$ constitutive strains can either be sensitive or insensitive to glucose repression (Charron and Michels, 1987; Gibson et al., 1997; Higgins et al., 1999).

Regarding the utilization of maltotriose by $S$. cerevisiae, little is known about its transport and 
regulation compared to the level of molecular detail described for the maltose transporter in yeast cells. Maltotriose is transported by a different transport system from the well-characterized maltose transport carried out through MALxl permeases. The AGT1 permease, which is able to transport not only maltotriose, but also maltose, trehalose and sucrose (Han et al., 1995; Stambuk et al., 1999; 2000; Stambuk and de Araújo, 2001; Zastrow et al., 2000; 2001; Alves et al., 2007), is found in a mallg locus on chromosome VII of $S$. cerevisiae. It is probably regulated through the same regulatory mechanisms as the others $M A L$ genes, since it has an $\mathrm{UAS}_{\mathrm{MAL}}$ sequence at its promoter region (Han et al., 1995; Alves et al., 2007), although it was recently shown that some industrial yeast strains may have divergent sequences at the promoter region of their $A G T 1$ gene, which have been shown not to be induced by maltose (Vidgren et al., 2011).

Some reports have recently suggested that the expression of $\alpha$-glucoside transporters in yeasts is higher in maltotriose-grown cells than in maltose-grown cells (Dietvorst et al., 2005; Salema-oom et al., 2005; Alves et al., 2007; 2008). Thus, due to the relevance of maltose and maltotriose transport in yeasts for the development of biotechnological processes that depend on the efficient fermentation of starch hydrolysates, in the present study we have analyzed the expression of the genes that encode maltose and maltotriose transporters in order to better understand the uptake of these $\alpha$-glucosides by $S$. cerevisiae cells.

\section{MATERIALS AND METHODS}

\section{Yeast Strains and Growth Conditions}

The $S$. cerevisiae strains used in the present study are described in Table 1. Cells were routinely grown on rich YP medium (1\% yeast extract and 2\% peptone) supplemented with $2 \%$ of glucose, maltose or maltotriose as the carbon source. The $\mathrm{pH}$ of each medium was adjusted to 5.0 with $\mathrm{HCl}$. Cells were grown aerobically at $28{ }^{\circ} \mathrm{C}$ with shaking $(160 \mathrm{rpm})$ in cotton-plugged Erlenmeyer flasks filled to $1 / 5$ of the volume with the medium. Cellular growth was followed by turbidity measurements at $570 \mathrm{~nm}$.

\section{a-Glucosidase Assays}

The $\alpha$-glucosidase activity in yeast cells collected at the exponential phase of growth was determined in situ with permeabilized cells as described previously (Stambuk, 1999) using $100 \mathrm{mM}$ maltose or maltotriose in $100 \mathrm{mM}$ MOPS (morpholinepropanesulfonic acid)- $\mathrm{NaOH}$ (pH 6.8) buffer. The glucose produced during maltose or maltotriose hydrolysis was determined by the glucose oxidase and peroxidase method using a commercial kit (BioDiagnosticaLaborclin). All assays were done in triplicate, and controls with previously boiled yeast cells were used. All activities were expressed as nmol of glucose produced by hydrolysis per milligram (dry weight) of cells per minute.

Table 1: S. cerevisiae strains and oligonucleotides used in this study.

\begin{tabular}{|l|l|c|}
\hline $\begin{array}{l}\text { Yeast strains } \\
\text { and primers }\end{array}$ & Relevant genotype or description & $\begin{array}{c}\text { Source or } \\
\text { reference }\end{array}$ \\
\hline Yeast strains: & & Alves et al. (2008) \\
1403-7A & MATa mal13 AGT1 MAL12 mal33 MAL31 MAL32 MAL4 gal3 gal4 trp1 ura3 suc $^{-}$ & Alves et al. (2008) \\
CEN.PK2-1C & MATa mal13 AGT1 MAL12 MAL2-8c SUC2 ura3-52 his341 leu2-3,112 trp1-289 \\
Primers: & & IDT \\
AGT1-1347F & TGGTGGAATGGGTTTTGGTT & IDT \\
AGT1-1397R & CCACCGGCACCATTACTAGC & IDT \\
MALx1-1352F & CTCATGGCGCTAAAATGGGT & IDT \\
MALx1-1403R & AAGAACGCGACAACCATTAGAAG & IDT \\
MPH2-1615F & ATCTGGGCTGTGGTTGACCTA & IDT \\
MPH2-1665R & TTCCACGAAAGTCTTTCCGG & IDT \\
ACT1-904F & GGTACCACCATGTTCCCAGG & IDT \\
ACT1-962R & GCCAAAGCGGTGATTTCCTT & \\
\hline
\end{tabular}




\section{Transport Assays}

Since yeast $\alpha$-glucoside transporters are $\mathrm{H}^{+}$-symporters with a known stoichiometry of one proton cotransported with each sugar molecule (Serrano, 1977; Stambuk et al., 1996; 1998), the rates of active $\mathrm{H}^{+}$-maltose or $\mathrm{H}^{+}$-maltotriose symport were determined as previously described (Stambuk and de Araújo, 2001; Alves et al., 2008) using a PHM84 research pHmeter attached to a TT1 Servograph (Radiometer, Copenhagen). Cells were suspended at a cellular density of about $12-15 \mathrm{~g}$ (dry weight) $\mathrm{L}^{-1}$ in water, without any buffer, and placed in a conical water-jacketed vessel in a total volume of $3 \mathrm{~mL}$. The suspension was mixed with a magnetic stirrer and the temperature of the circulating water was regulated at $30{ }^{\circ} \mathrm{C}$. The $\mathrm{pH}$ of the cellular suspension was adjusted to 5.0 with $\mathrm{HCl}$, and the initial rates of sugar-induced proton uptake were calculated from the slope of the initial $(<10 \mathrm{~s})$ part of the curve obtained on the recorder by subtracting the basal rate of proton uptake observed before the addition of 0.3 to $100 \mathrm{mM}$ of the sugar. To calculate the rate of $\mathrm{H}^{+}$uptake, a calibration curve was obtained by addition of 50-100 nmol of $\mathrm{HCl}$ to the cell suspension. Kinetic constants were obtained as previously described (Stambuk et al., 2000; Stambuk and de Araujo, 2001). Experimental points are represented by the symbols used in the figures while lines represent the best fit obtained for the data set. All activities were expressed as nmol of substrate transported per milligram (dry weight) of cells per minute.

\section{Quantitative RT-PCR (qRT-PCR)}

Yeast cells were grown on rich media supplemented with $2 \%$ glucose, maltose or maltotriose, collected, rapidly frozen in liquid nitrogen, and stored at $-80{ }^{\circ} \mathrm{C}$. The total RNA was extracted with TRIzol (Invitrogen), and $1 \mu \mathrm{g}$ of extracted RNA was treated with DNAse (Roche) and used for cDNA synthesis with a Reverse Transcription System kit
(Promega). For real-time PCR, the KAPA SYBR FAST qPCR kit was used with 20 ng of cDNA, according to the manufacturer's instructions. The pairs of primers AGT1-1347F and AGT1-1397R; MALx1-1352F and MALx1-1403R; MPH2-1615F and MPH2-1665R, and ACT1-904F and ACT1-962R (Table 1) were used to evaluate the expression of the $A G T 1, M A L x 1, M P H 2 / M P H 3$ and $A C T 1$ genes, respectively. The reaction was performed with a StepOnePlus Real-Time PCR System (Applied Biosystem). A dissociation curve was generated for each assay in order to confirm the amplification of only one product. All samples were tested in triplicate and the results presented are means and standard deviation obtained from two independent experiments. The expression values represent a relative quantification obtained by dividing the amount of mRNA from each analyzed gene by the mRNA from the house-keeping gene $A C T 1$.

\section{RESULTS AND DISCUSSION}

The two yeast strains analyzed in the present work (strains 1403-7A and CEN.PK2-1C) have unrelated genetic backgrounds, but all are able to grow on, consume and ferment both maltose and maltotriose efficiently (Alves et al., 2008). These strains have in their genomes not only the AGT1 and MPH2/MPH3 genes, but also at least two copies of MALxl genes: MAL21 and MAL31 (both strains) and, in the case of strain 1403-7A, also the MAL41 gene (Alves et al., 2008; Duval et al., 2010). The results presented in Table 2 indicate that, in both strains, the activity of $\alpha$-glucoside transporters is higher in maltotriose-grown cells than in maltosegrown cells, as already described in the literature (Dietvorst et al., 2005; Salema-oom et al., 2005; Alves et al., 2007; 2008), while the $\alpha$-glucosidase activity is practically the same in cells of each strain grown on either carbon source, except for maltotriose hydrolysis by strain 1403-7A, which was two-fold higher in maltose-grown cells.

Table 2: Maltose and maltotriose active transport and hydrolysis by yeast strains.

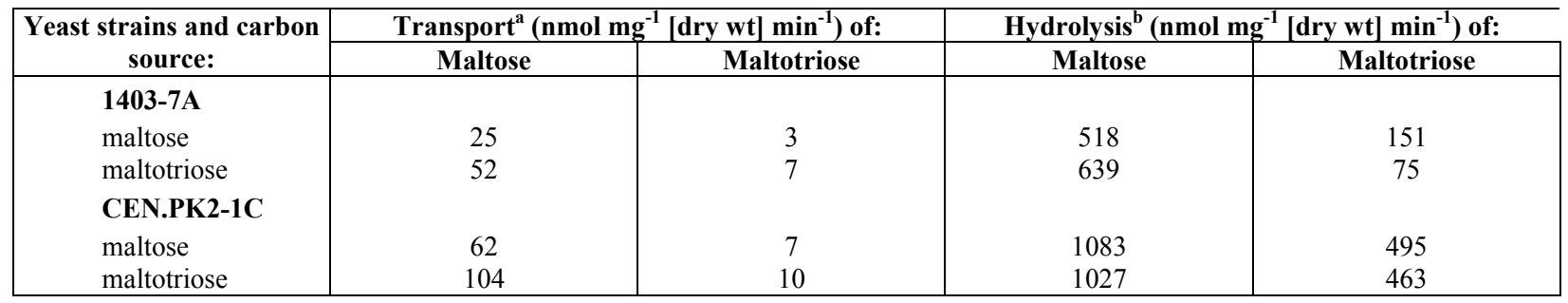

${ }^{a}$ Estimated by the rates of $\mathrm{H}^{+}$-cotransport by the yeast cells using $5 \mathrm{mM}$ final sugar concentration.

Assays were carried out in triplicate, with standard errors of less than $15 \%$.

${ }^{b}$ Determined with permeabilized yeast cells using $100 \mathrm{mM}$ final sugar concentration. Assays

were carried out in triplicate, with standard errors of less than $10 \%$ 
Since an increase in transport activity could be the consequence of a change in affinity of the transporter(s) present in the plasma membrane (Smit et al., 2007), we performed a kinetic analysis of the active maltotriose- $\mathrm{H}^{+}$symport activity by these strains after growth on maltose or maltotriose (Figure 1). These two strains have a low affinity $\left(K_{\mathrm{m}} 36 \pm 2 \mathrm{mM}\right)$ maltotriose- $\mathrm{H}^{+}$symport activity, and the only difference between maltose-grown and maltotriosegrown cells is the higher transport capacity of the yeast cells grown in this last carbon source (Figure 1, $\mathrm{A}$ and $\mathrm{B})$.

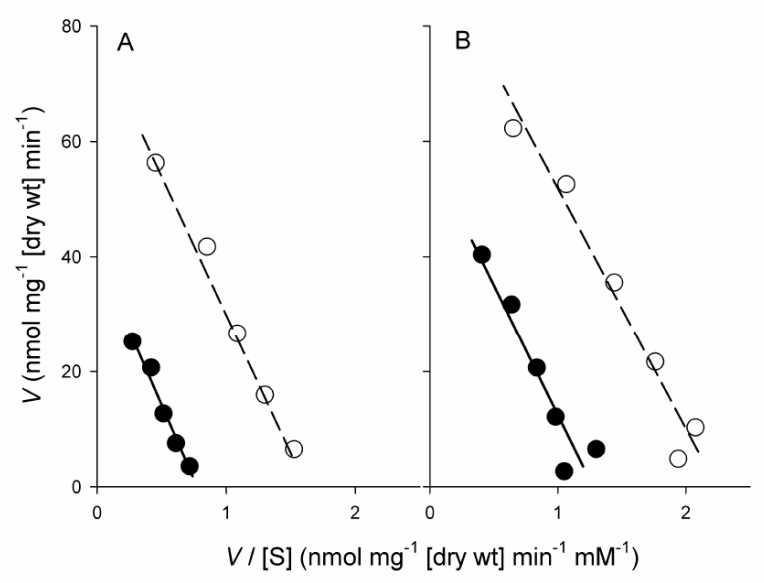

Figure 1: Kinetics of active maltotriose- $\mathrm{H}^{+}$symport by yeast cells. Eadie-Hofstee plots of $\mathrm{H}^{+}$cotransport during maltotriose uptake by maltose-grown (filled symbols) or maltotriose-grown (open symbols) cells of strain 1403-7A (A) or CEN.PK2-1C (B), determined as described in Materials and Methods.

This relatively low affinity for maltotriose is a known characteristic of the $A G T 1$ permease, indicating that this transporter is probably responsible for maltotriose uptake in both strains. In order to better characterize the reason for these results, we quantified the expression of mRNA transcripts from the MALx1, MPH2/MPH3 and AGT1 genes through quantitative RT-PCR (qRT-PCR). To this end, the yeast strains were grown on rich media containing $2 \%$ glucose, maltose or maltotriose, and cells were collected at the middle of the exponential phase of growth for subsequent RNA extraction.

Figure 2 (parts $\mathrm{A}$ and $\mathrm{B}$ ) shows that, when the $M A L$-constitutive strains 1403-7A and CEN.PK2-1C are metabolizing maltotriose, the expression levels of the $A G T 1$ and MALxl genes are similar to the ones observed in maltose growing cells, indicating that the higher transport activity found in maltotriose-growing cells is not a consequence of higher induction of $M A L$ genes by this carbon source. Figure 2 also shows that, while strain 1403-7A is not repressed by glucose, strain CEN.PK2-1C was fully repressed by this monosaccharide. Our results are in agreement with previous characterization of the MALx3 regulators, which indicated that the $M A L 43^{\mathrm{C}}$ mutant allele is insensitive to glucose repression (Charron and Michels, 1987; Wang and Needleman, 1996), while the constitutive $M A L 23-8^{\mathrm{C}}$ regulator present in the CEN.PK2 strains is glucose repressible (Rodicio and Zimmermann, 1984; Kopetzki et al., 1989). In agreement with the above results, there was also no difference in the expression levels of $M P H 2 / M P H 3$ genes between maltose- and maltotriose-grown cells (Figure 2C). It is interesting to note, however, that although it has been suggested that these genes have their expression induced by MALx3 regulators (Day et al., 2002), our results showed no repression of such genes by glucose, indicating that expression of MPH2/MPH3 genes is probably not controlled exactly like the MALXl or $A G T 1$ genes.
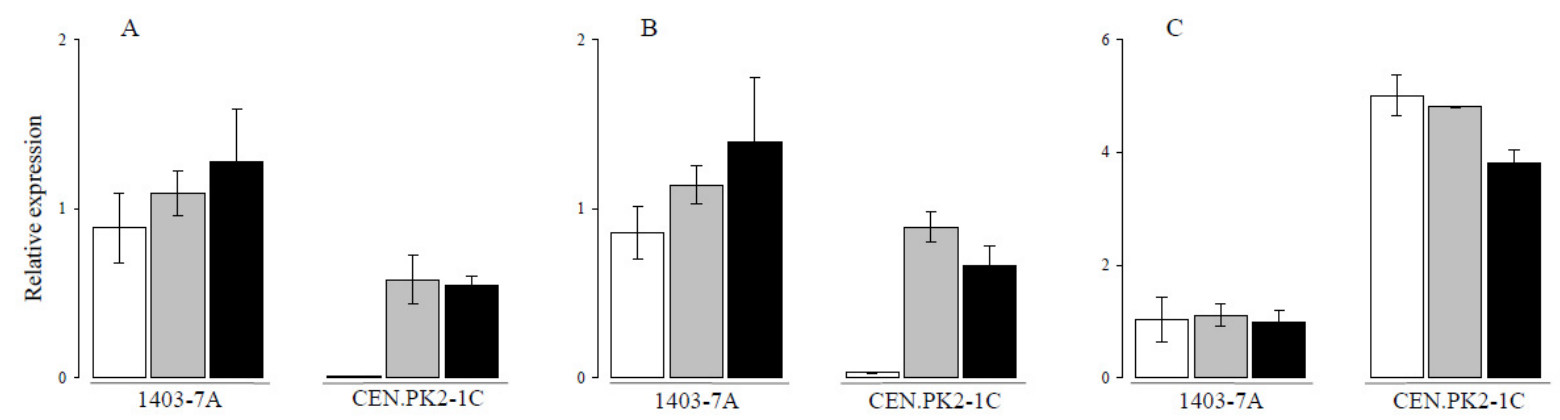

Figure 2: Relative expression of the $A G T 1$ and $M A L x 1$ genes. After reaching the exponential growth phase on rich media supplemented with glucose (white bars), maltose (grey bars) or maltotriose (black bars), the cells were harvested for RNA extraction and relative quantification (in comparison to $A C T 1$ ) of the $A G T 1$ (A), MALxl (B) and $M P H 2 / M P H 3(C)$ transcripts present in strain 1403-7A and CEN.PK2-1C, performed as described in Materials and Methods. 
While the kinetic parameters of sugar transport are a property of the transporter protein, the transport capacity $\left(V_{\max }\right)$ depends upon how many transporters are expressed and present (in an active form) in the cell membrane. Unfortunately studies using a $M A L^{\mathrm{C}}$ strain with its $A G T 1$ permease tagged with greenfluorescent-protein (GFP) were also inconclusive regarding differences in the expression of the protein by the cells grown on maltose or maltotriose (data not shown). Probably other factors, including posttranslational regulation of the transporters (Hu et al., 2000; Jiang et al., 2000; Gadura et al., 2006), or in the case of active transporters, also the energetic state of the cells (Guimarães and Londesborough, 2008; Guimarães et al., 2008), can explain the differences in transport rates observed in these $M A L^{\mathrm{C}}$ yeast strains. Since both the repression and catabolic inactivation of transporters is trigged not only by external glucose, but also by glucose molecules generated inside the cells due to high rates of maltose hydrolysis (Hu et al., 1995; 2000; Gibson et al., 1997; Jiang et al., 1997; 2000), our results of lack of correlation between the expression of the genes and the transport activity measured in the cells could also be explained by the different rates of $\alpha$-glucoside hydrolysis by the yeast cells, where maltose hydrolysis is, at least, two-fold higher than maltotriose hydrolysis (see Table 2 and Zastrow et al., 2000; 2001; Alves et al., 2007; 2008), thus generating more glucose molecules during growth in maltose, when compared with growth on maltotriose.

\section{ACKNOWLEDGMENTS}

This work was supported in part by grants from the Brazilian agencies CNPq and CAPES. We thank R. Needleman (Wayne State University) and A. Kruckeberg (University of Amsterdam) for providing yeast strains, and $\mathrm{H}$. Tournu for technical collaboration during the real time PCR experiments.

\section{REFERENCES}

Alves, S. L. Jr., Herberts, R. A., Hollatz, C., Miletti, L. C. and Stambuk, B. U., Maltose and maltotriose active transport and fermentation by Saccharomyces cerevisiae. Journal of the American Society of Brewing Chemistry, 65, 99-104 (2007).

Alves, S. L. Jr., Herberts, R. A., Hollatz, C., Trichez, D., Miletti, L. C., de Araujo, P. S. and Stambuk, B. U., Molecular analysis of maltotriose active transport and fermentation by Saccharomyces cerevisiae reveals a determinant role for the $A G T 1$ permease. Applied and Environmental Microbiology, 74, 1494-1501 (2008).

Charron, M. J. and Michels, C. A., The constitutive glucose-repression-insensitive mutation of the yeast MAL4 locus is an alteration of the MAL3 gene. Genetics, 116, 23-31 (1987).

Day, R. E., Higgins, V. J., Rogers, P. J. and Dawes, I. W., Characterization of the putative maltose transporters encoded by YDL247w and YJR160c. Yeast, 19, 1015-1027 (2002).

Dietvorst, J., Londesborough, J. and Steensma, H. Y., Maltotriose utilization in lager yeast strains: MTT1 encodes a maltotriose transporter. Yeast, 22, 775-788 (2005).

Duval, E. H., Alves, S. L. Jr., Dunn, B., Sherlock, G. and Stambuk, B. U., Microarray karyotyping of maltose-fermenting Saccharomyces yeasts with differing maltotriose utilization profiles reveals copy number variation in genes involved in maltose and maltotriose utilization. Journal of Applied Microbiology 109, 248-259 (2010).

Gadura, N., Robinson, L. C. and Michels, C. A., Glc7-Reg1 Phosphatase signals to Yck1,2 Casein Kinase 1 to regulate transport activity and glucoseinduced inactivation of Saccharomyces maltose permease. Genetics, 172, 1427-1439 (2006).

Gibson, A. W., Wojciechowicz, L. A., Danzi, S. E., Zhang, B., Kim, J. H., Hu, Z. and Michels, C. A., Constitutive mutations of the Saccharomyces cerevisiae MAL-activator genes MAL23, MAL43, MAL63, and mal64. Genetics, 146, 1287-1298 (1997).

Guimarães, P. M. and Londesborough, J., The adenylate energy charge and specific fermentation rate of brewer's yeasts fermenting high- and very high-gravity worts. Yeast, 25, 47-58 (2008).

Guimarães, P. M., Multanen, J. P., Domingues, L., Teixeira, J. A., and Londesborough, J., Stimulation of zero-trans rates of lactose and maltose uptake into yeasts by preincubation with hexose to increase the adenylate energy charge. Applied and Environmental Microbiology, 74, 3076-3084 (2008).

Han, E. K., Cotty, F., Sottas, C., Jiang, H. and Michels, C. A., Characterization of $A G T 1$ encoding a general $\alpha$-glucoside transporter from Saccharomyces. Molecular Microbiology, 17, 1093-1107 (1995).

Higgins, V. J., Braidwood, M., Bell, P. J. L., Bissinger, P., Dawes, I. W. and Attfield, P. V., Genetic evidence that noninduced maltase and maltose permease activities, governed by MALx3encoded transcriptional regulators, determine efficiency of gas production by baker's yeast in 
unsugared dough. Applied and Environmental Microbiology, 65, 680-685 (1999).

Hu, Z., Nehlin, J. O., Ronne, H. and Michels, C. A., $M I G 1$-dependent and MIG1-independent glucose regulation of MAL gene expression in Saccharomyces cerevisiae. Current Genetics, 28, 258-266 (1995).

Hu, Z., Yue, Y., Jiang, H., Zhang, B., Sherwood, P. W. and Michels, C. A., Analysis of the mechanism by which glucose inhibits maltose induction of MAL gene expression in Saccharomyces. Genetics, 154, 121-132 (2000).

Jiang, H., Medintz, I. and Michels, C. A., Two glucose sensing/signaling pathways stimulate glucoseinduced inactivation of maltose permease in Saccharomyces. Molecular Biology of the Cell, 8, 1293-1304 (1997).

Jiang, H., Medintz, I., Zhang, B. and Michels, C. A., Metabolic signals trigger glucose-induced inactivation of maltose permease in Saccharomyces. Journal of Bacteriology, 182, 647-654 (2000).

Kim, J. and Michels, C. A., The MAL63 gene of Saccharomyces encodes a cysteine-zinc finger protein. Current Genetics, 14, 319-323 (1988).

Kodama, Y., Fukui, N., Ashikari, T., Shibano, Y., Morioka-Fujimoto, K., Hiraki, Y. and Nakatani, $\mathrm{K}$., Improvement of maltose fermentation efficiency: Constitutive expression of $M A L$ genes in brewing yeasts. Journal of the American Society of Brewing Chemistry, 53, 24-29 (1995).

Kopetzki, E., Zellner, E., Schumacher, G. and Zimmermann, F. K., Nucleotide sequence of the Saccharomyces cerevisiae positive regulatory mutant gene $M A L 2-8^{\mathrm{c}} p$. Nucleic Acid Research, 17, 5390 (1989).

Levine, J., Tanouye, L. and Michels, C. A., The $\mathrm{UAS}_{\mathrm{MAL}}$ is bidirecional promoter element required for the expression of both the MAL61 and MAL62 genes of the Saccharomyces cerevisiae. Current Genetics, 22, 181-189 (1992).

Medintz, I., Jiang, H., Han, E. K., Cui, W., and Michels, C. A., Characterization of the glucoseinduced inactivation of maltose permease in Saccharomyces cerevisiae. Journal of Bacteriology, 178, 2245-2254 (1996).

Michels, C. A. and Needleman, R. B., The dispersed, repeated family of MAL loci in Saccharomyces spp. Journal of Bacteriology, 157, 949-952 (1984).

Naumov, G. I., Naumova, E. S. and Michels, C. A., Genetic variation of the repeated $M A L$ loci in natural population of Saccharomyces cerevisiae and Saccharomyces paradoxus. Genetics, 136, 803-812 (1994).

Needleman, R. B., Control of maltase synthesis in yeast. Molecular Microbiology, 5, 2079-2084 (1991).

Needleman, R. B., Kaback, D. B., Dubin, R. A., Perkins, E. L., Rosenberg, N. G., Sutherland, K. A., Forrest, D. B. and Michels, C. A., MAL6 of Saccharomyces: A complex genetic locus containing three genes required for maltose fermentation. Proceedings of National Academy of Science, 81, 2811-2815 (1984).

Novak. S., Zechner-Krpan, V. and Marié, V., Regulation of maltose transport and metabolism in Saccharomyces cerevisiae. Food Technology and Biotechnology, 42, 213-218 (2004).

Querol, A. and Bond, U., The complex and dynamic genomes of industrial yeasts. FEMS Microbiology Letters, 293, 1-10 (2009).

Rautio, J. and Londesborough, J., Maltose transport by brewer's yeasts in brewer's wort. Journal of the Institute of Brewing, 109, 251-261 (2003).

Riballo. E., Herwijer, M., Wolf, D. H. and Lagunas, R., Catabolite inactivation of the yeast maltose transporter occurs in the vacuole after internalization by endocytosis. Journal of Bacteriology, 177, 5622-5627 (1995).

Rodicio, R. and Zimmermann, F. K., Cloning of maltase regulatory genes in Saccharomyces cerevisiae. 1. Isolation of the $M A L 2-8^{c}$ regulatory gene. Current Genetics, 9, 539-545 (1984).

Salema-Oom, M., Pinto, V. V., Gonçalves, P. and Spencer-Martins, I., Maltotriose utilization by industrial Saccharomyces strains: Characterization of a new member of the $\alpha$-glucoside transporter family. Applied and Environmental Microbiology, 71, 5044-5049 (2005).

Santangelo, G. M., Glucose signaling in Saccharomyces cerevisiae. Microbiology and Molecular Biology Reviews, 70, 253-282 (2006).

Serrano, R., Energy requirements for maltose transport in yeasts. European Journal of Biochemistry, 80, 97-102 (1977).

Sirenko, O. I., Ni, B. and Needleman, R. B., Purification and biding properties of Mal63p activator of Saccharomyces cerevisiae. Current Genetics, 27, 509-516 (1995).

Smit, A., Cordero-Otero, R. R. and Pretorius, I. S., Differences among $A G T 1$-encoded $\alpha$-glucoside transporters and their ability to transport maltotriose in Saccharomyces yeasts. Annals of Microbiology, 57, 77-84 (2007).

Stambuk, B. U., A simple experiment illustrating metabolic regulation: induction versus repression of yeast $\alpha$-glucosidase. Biochemical Education, 27, 177-180 (1999).

Stambuk, B. U., Batista, A. S. and de Araújo, P. S., 
Kinetics of active sucrose transport in Saccharomyces cerevisiae. Journal of Bioscience and Bioengineering, 89, 212-214 (2000).

Stambuk, B. U., da Silva, M. A., Panek, A. D. and de Araújo, P. S., Active $\alpha$-glucoside transport in Saccharomyces cerevisiae. FEMS Microbiology Letters, 170, 105-110 (1999).

Stambuk, B. U., de Araujo, P. S., Panek, A. D., and Serrano, R., Kinetics and energetics of trehalose transport in Saccharomyces cerevisiae. European Journal of Biochemistry, 237, 876-881 (1996).

Stambuk, B. U. and de Araújo, P. S. Kinetics of active $\alpha$-glucoside transport by Saccharomyces cerevisiae. FEMS Yeast Research, 1, 73-78 (2001).

Stambuk, B. U., Panek, A. D., Crowe, J. H., Crowe, L. M. and de Araújo, P. S., Expression of highaffinity trehalose- $\mathrm{H}^{+}$symport in Saccharomyces cerevisiae. Biochimica et Biophysica Acta, 1379, 118-128 (1998).

Vidgren, V., Kankainen, M., Londesborough, J. and Ruohonen, L., Identification of regulatory elements in the $A G T 1$ promoter of ale and lager strains of brewer's yeast. Yeast, 28, 579-594 (2011).

Wang, J. and Needleman, R. B., Removal of a Miglp binding site converts a MAL63 constitutive mutant derived by interchromossomal gene con- version to glucose insensitivity. Genetics, 142, 51-63 (1996).

Wang, X., Bali, M., Medintz, I. and Michels, C. A., Intracellular maltose is sufficient to induce $M A L$ gene expression in Saccharomyces cerevisiae. Eukariotic Cell, 1, 696-703 (2002).

Willaert, R., Sugar consumption kinetics by brewer's yeast during the primary beer fermentation. Cerevisia, 26, 43-49 (2001).

Yoon, S. H., Mukerjea, R. and Robyt, J. F., Specificity of yeast (Saccharomyces cerevisiae) in removing carbohydrates by fermentation. Carbohydrate Research, 338, 1127-1132 (2003).

Zastrow, C. R., Hollatz, C., de Araujo, P. S. and Stambuk, B. U., Maltotriose fermentation by Saccharomyces cerevisiae. Journal of Industrial Microbiology and Biotechnology, 27, 34-38 (2001).

Zastrow, C. R., Mattos, M. A., Hollatz, C. and Stambuk, B. U., Maltotriose metabolism by Saccharomyces cerevisiae. Biotechnology Letters, 22, 455-459 (2000).

Zheng, X., D'Amore, T., Russell, I. and Stewart, G. G., Factors influencing maltotriose utilization during brewery wort fermentations. Journal of the American Society of Brewing Chemistry, 52, 4147 (1994). 